\title{
Neonatal Schwartz-Jampel syndrome: a common autosomal recessive syndrome in the United Arab Emirates
}

\author{
L I Al-Gazali, M Varghese, E Varady, J Al Talabani, J Scorer, D Bakalinova
}

Department of Paediatrics, Faculty of Medicine and Health Sciences, UAE

University, PO Box

17666, Al Ain, United

Arab Emirates

L I Al-Gazali

Department of Paediatrics, Oasis

Hospital, Al Ain, UAE $M$ Varghese

\section{Department of}

Paediatrics, Corniche

Hospital, Abu Dhabi,

UAE

J Al Talabani

Department of Paediatrics, Tawam Hospital, Al Ain, UAE E Varady

J Scorer

\section{Department of}

Radiology, Faculty of

Medicine and Health

Sciences, UAE

University, Al Ain,

UAE

D Bakalinova

Correspondence to: Dr Al Gazali.

Received 5 June 1995 Revised version accepted for publication 26 October 1995

\begin{abstract}
Schwartz-Jampel syndrome (SJS) is a heterogeneous autosomal recessive syndrome of myotonia and bone dysplasia. Two types have been recognised: the classical type with late infantile or childhood manifestation and a rarer form with neonatal manifestation.

We report five families with a total of 11 children affected with severe neonatal SJS. All presented after birth with skeletal abnormalities and feeding difficulties. Five had the typical pursed appearance of the mouth. Nine died from respiratory complications (five in the neonatal period and four before 2 years of age). One ( 4 months old) remains hospitalised since birth requiring continuous oxygen supplementation and one ( 5 months old) requires nasogastric tube feeding and has repeated attacks of aspiration.

Only seven of the 17 previously reported neonatal SJS cases had a similar course to the patients in this report. We suggest that within neonatal SJS there is a subgroup which manifests severe respiratory and feeding problems and has a poor prognosis. This report brings the total number of children with neonatal SJS reported from the UAE to 14. This represents the largest review of this syndrome to date from one centre and indicates that this syndrome is fairly common in the population of the UAE.

(f Med Genet 1996;33:203-211)
\end{abstract}

Key words: Schwartz-Jampel syndrome; United Arab Emirates; autosomal recessive.

Schwartz-Jampel syndrome (SJS) is characterised by myotonia with mask-like face and skeletal dysplasia with growth retardation. Two types have been described. The classical type which usually manifests late in infancy and a rarer, more severe form with neonatal manifestation. Although 17 cases of SJS with neonatal manifestation have been reported, ${ }^{1-11}$ the clinical and radiological features in the neonatal period of this type of SJS have not been properly delineated, mainly because in the majority of the reported cases the diagnosis was not suspected until after the neonatal period.

In this paper we report five families with a total of 11 children affected with severe neonatal SJS. The clinical and radiological features of previously reported cases of SJS with neonatal manifestation will be reviewed.

\section{Case reports}

FAMILY 1

The family shows complex consanguinity with a total of four affected out of 12 children in two sibships. There was one miscarriage in each sibship (fig 1).

\section{Case 1 (IV.7)}

This female was the product of a normal pregnancy and term delivery. Birth weight was $2500 \mathrm{~g}$ (5th centile) and length was $45 \mathrm{~cm}$ $(<5$ th centile). At birth she was noted to have the following dysmorphic features: asymmetrical head, short palpebral fissures, pursed appearance of the mouth during crying or any stimulation, and abnormal ear helices. There was bilateral camptodactyly with overlapping fingers. All the limbs appeared short. There was limited extension of the elbow joints, bowing of the femur and tibia, and overlapping toes (fig 2A, table)

$X$ ray at 2 days of age showed bowing of the long bones, particularly of the lower limbs, with cortical thickening of the diaphyseal concavities and osteoporosis. There was metaphyseal flaring of the long bones (fig $2 \mathrm{~B}$, table).

The baby continued to need oxygen therapy, although clinically and radiologically the chest and heart were normal. She died suddenly at the age of 10 days.

\section{Case $2(I V \cdot 5)$}

Data were retrieved from hospital records. A boy, the brother of case 1 , was the product of a normal pregnancy and breech delivery at term. The baby was asphyxiated at birth and needed resuscitation with ventilatory support.

He was noted to have shortening and bowing of the lower limbs. A few days after birth, cardiomegaly was diagnosed, which was attributed to the asphyxia and was treated accordingly. He later developed pneumonia and died on the eleventh day of life from respiratory complications.

\section{Case $3(V \cdot 8)$}

This boy was born at 36 weeks' gestation by normal vaginal delivery. Birth weight was $1980 \mathrm{~g}$ (10th centile), length was $46 \mathrm{~cm}$ ( $>10$ th 
।

II

III

IV

V

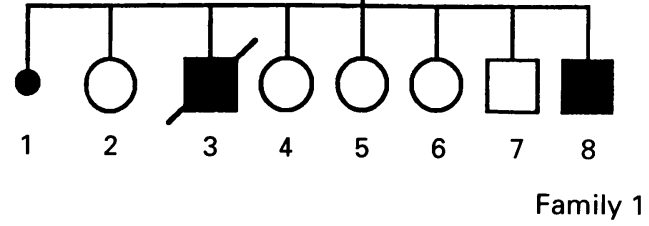

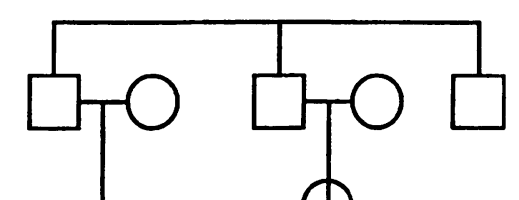

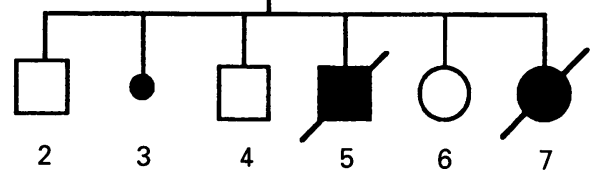

III

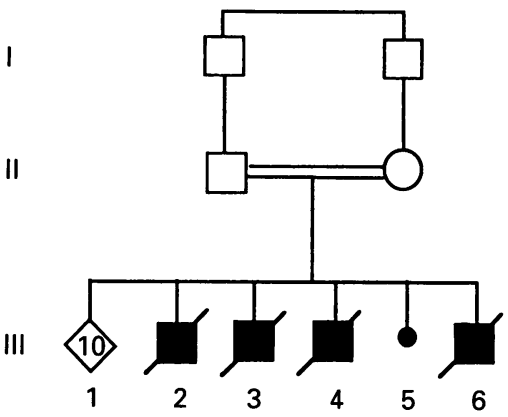

Family 3

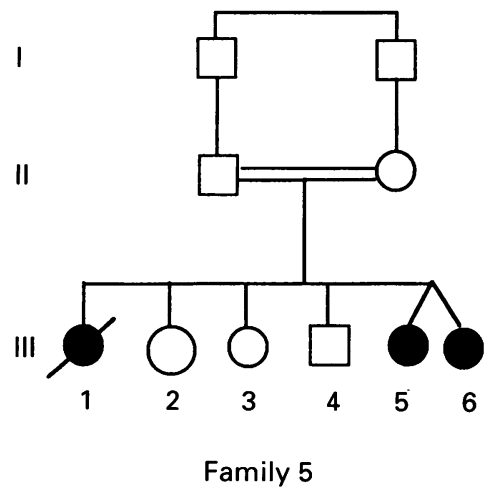

Figure 1 Pedigrees of the five families.

centile), and head circumference was $31.5 \mathrm{~cm}$ ( $>10$ th centile).

At birth he was noted to have the following dysmorphic features: short palpebral fissures, bilateral camptodactyly with overlapping fingers, limited extension of the elbows, shortening and bowing of the lower extremities, particularly the tibia, and bilateral positional talipes equinovarus (fig 3A). He was also noted to have a pursed appearance of the mouth during crying.

Immediately after birth he developed respiratory difficulties and was ventilated with high pressure oxygen. On several occasions he 

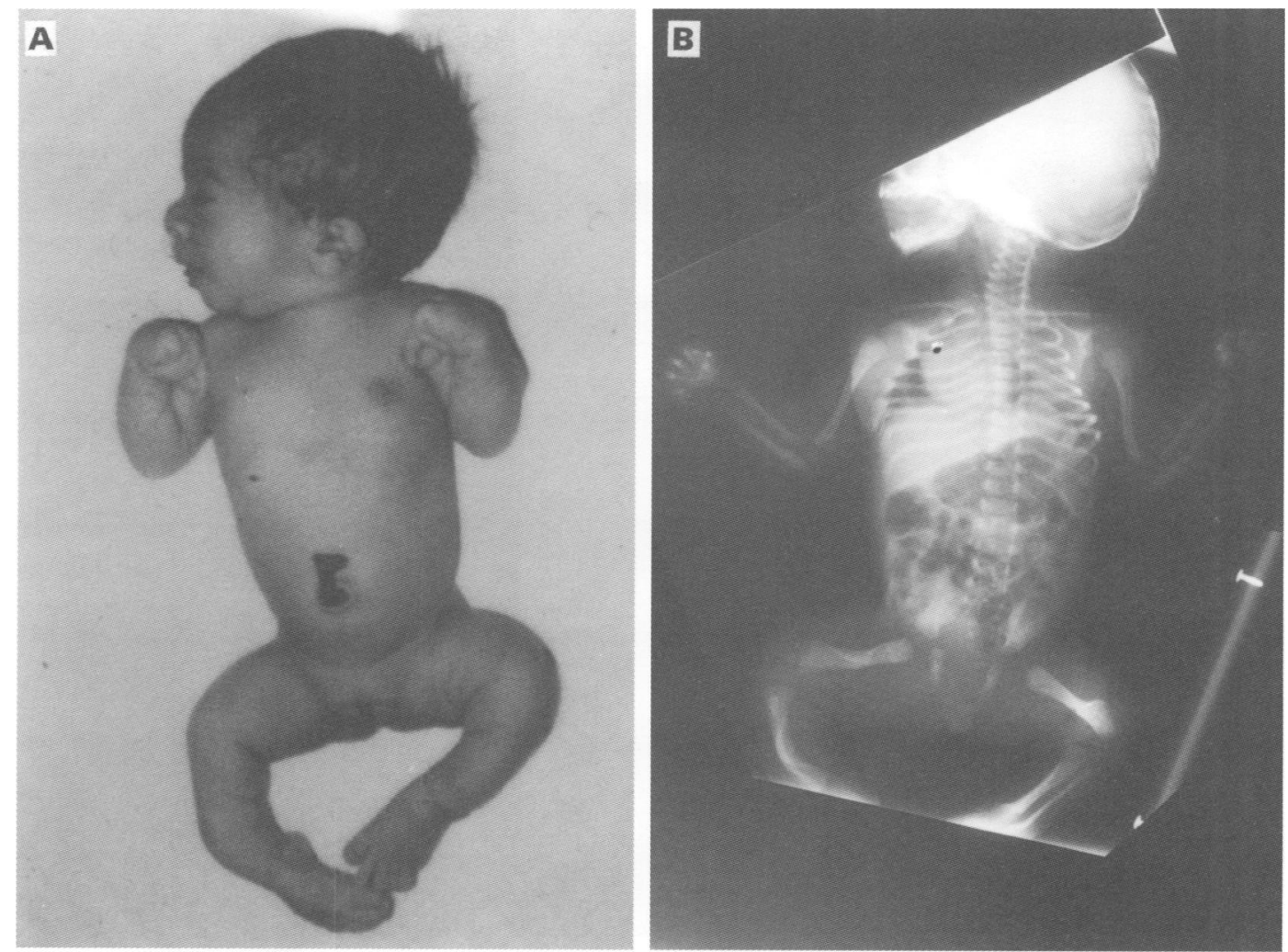

Figure 2 (A) General appearance of case 1, family 1. Note the shortening and bowing of the lower limbs and camptodactyly. (B) Total $x$ ray of case 1, family 1 showing the shortening and bowing of the long bones of the lower limbs with cortical thickening and the diaphyseal concavities.

had a spiking temperature although all investigations showed no evidence of sepsis. The baby continued to require supplemental oxygen after weaning him off the ventilator, failed to suck, and had to be fed by nasogastric tube.

Skeletal survey in the neonatal period showed generalised osteoporosis with wide, flared metaphyses. Lower limb bowing and cortical thickening in the diaphyseal concavities was noted (fig 3B). Epiphyseal development was appropriate for age. Skull and spine $x$ rays were normal (table).

EMG at 6 weeks showed continuous firing of complex discharges in all muscles tested. He had recurrent febrile episodes and pneumonias. At the age of 3 months he was still hospitalised requiring $60 \%$ box oxygen and nasogastric tube feeding.

Clinical and radiological features of 11 cases of neonatal SFS

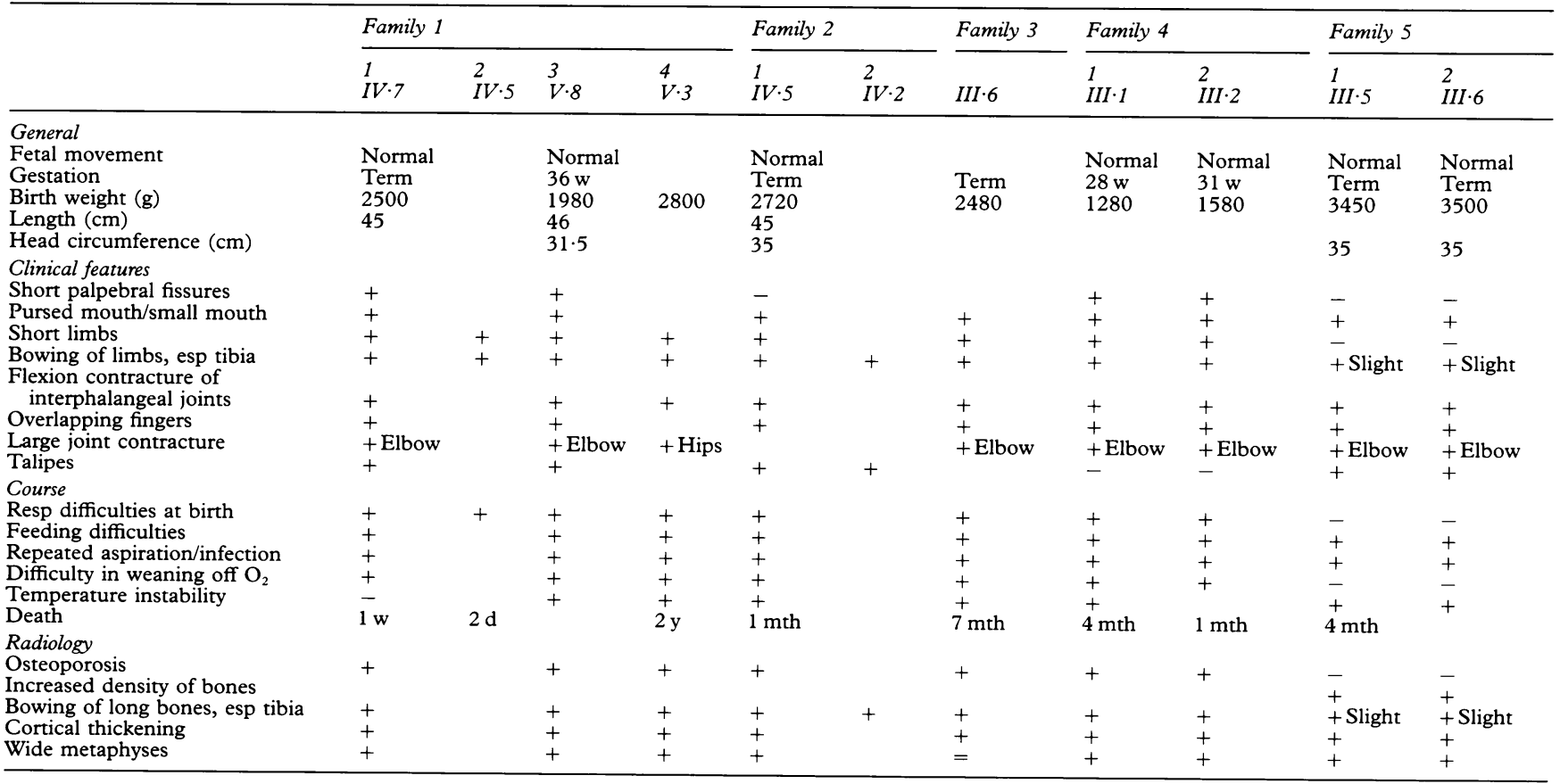




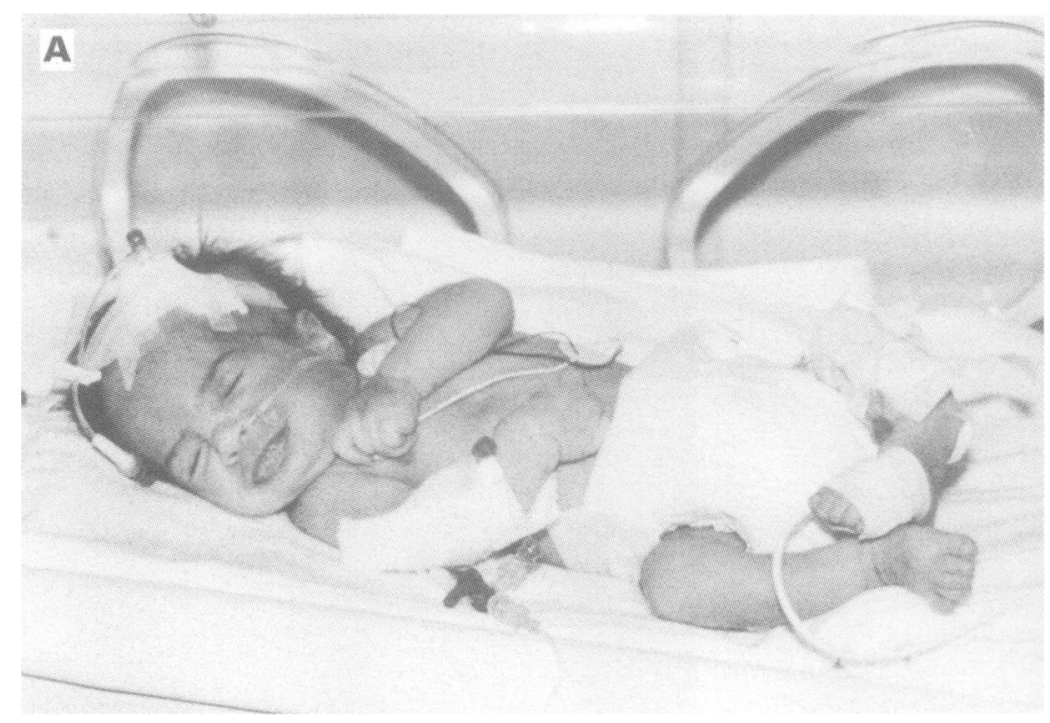

Figure 3 (A) General appearance of case 3, family 1. (B) X ray of the long bones of the lower limbs in case 3, family 1 showing osteoporosis at the metaphyses and bowing and cortical thickening at the diaphyses.

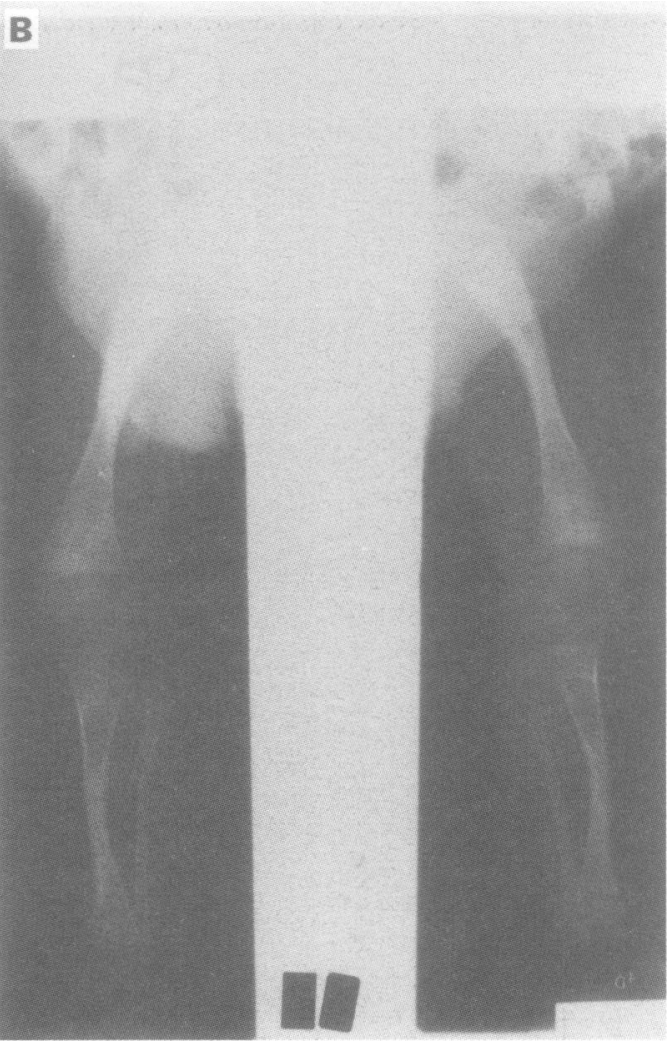

bone deformities and tracheo-oesophageal fistula for which he was operated upon. He died eight days after surgery from respiratory complications. No other information is available.

A boy, the brother of case 3 , was bo caesaerean section at 42 weeks' gestation because of malposition. He developed a high temperature the following day; however, blood cultures, CSF, and urine cultures were negative. The baby failed to suck either from the breast or bottle, but feeds by nasogastric tube were well tolerated.

Examination at 7 days of age showed a weight of $2.8 \mathrm{~kg}$ ( $>10$ th centile) and head circumference of $36.5 \mathrm{~cm}$ ( 50 th centile). The ears appeared low set and backward slanting. There were bilateral simian creases and flexion contractures of the third and fourth fingers bilaterally with ulnar deviation of the thumb. The lower limbs showed severely bowed tibiae and shorter than normal femora. There was limited abduction of the hips and a large inguinal hernia on the right side. Routine investigations were normal. Chest $x$ ray showed eventration of the right diaphragm. CT scans of the brain and chromosomes were normal. Skeletal survey at 1 month of age showed similar findings to case 3 (table).

The baby continued to have difficulty in feeding owing to his inability to suck. Although there was no obvious respiratory distress, his colour was generally pale and dusky. After discharge from hospital he needed repeated admissions because of recurrent pneumonia. $\mathrm{He}$ died at the age of 2 years from respiratory complications.

\section{FAMILY 2}

The parents are UAE nationals, first cousins once removed, and are of the same tribe as the first family (fig 1). Their first baby (IV-2) had

\section{Case $1(I V \cdot 5)$}

This boy was the product of a normal pregnancy. Delivery at term was by LCS because of placenta praevia. Birth weight was $2720 \mathrm{~g}$ (10th centile), length $45 \mathrm{~cm}$ ( $<5$ th centile), and head circumference $35 \mathrm{~cm}$ ( 50 th centile).

Examination at birth showed shortening of the limbs, particularly the lower limbs, with bowing of the femora and tibiae. There was bilateral camptodactyly, overlapping fingers, and bilateral talipes equinovarus. The baby had the typical pursed appearance of the mouth during crying, (fig $4 \mathrm{~A}, \mathrm{~B}$, table).

No other abnormalities were detected apart from a systolic murmur at the left sternal edge. Throughout his stay in hospital he had irregular fever although investigation for sepsis on many require $36 \%$ oxygen in order to maintain oxygen saturation of 95 to $97 \%$. He was unable to swallow and required nasogastric tube feeding.

Chromosome analysis, CT scan of the brain, investigations for sepsis, and blood chemistry were normal. Echocardiography showed a small ventricular septal defect. Skeletal $x$ rays at 2 days of age showed bowing of both femora and tibiae with cortical thickening at the diaphyseal concavities (fig 4C, table).

Muscle biopsy showed slightly atrophic type I and II skeletal muscle fibres in bundles separated by thickened fibrous septa and fatty infiltration. A few fibres were somewhat hyoccasions was negative. He also continued to 


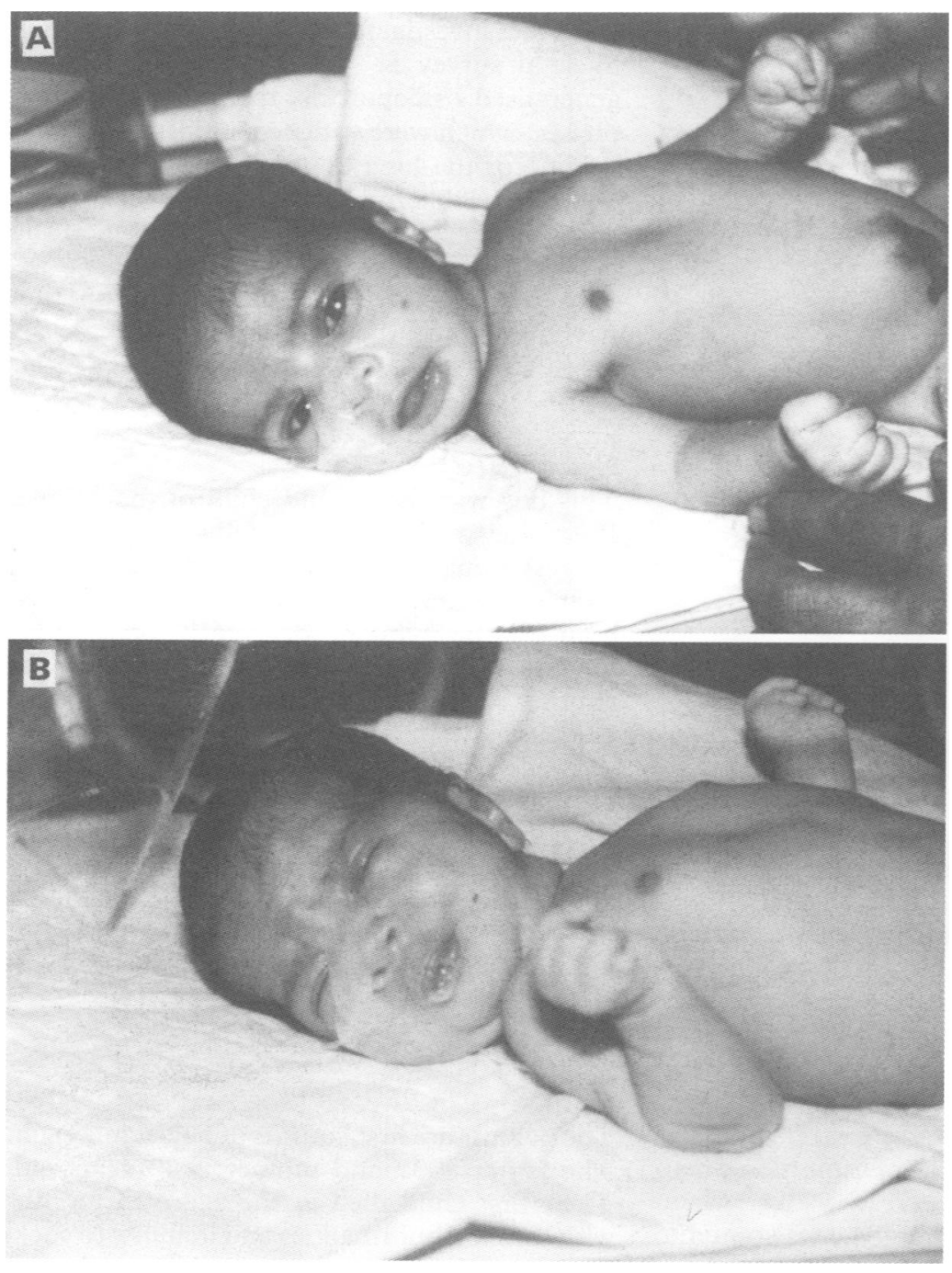

pertrophic. The muscle spindle, nerves, and blood vessels were normal.

The child needed continuous nasal oxygen therapy with a flow between 2 and $31 / \mathrm{min}$ and nasogastric tube feeding. He deteriorated slowly and died at 1 month of age.

\section{FAMILY 3}

The parents are first cousins of Omani origin. There is a history of one miscarriage and three other babies died in the neonatal period with similar abnormalities. The other 10 children are said to be normal (fig 1).

\section{Case 1 (III.6)}

This boy was the product of a normal pregnancy and term delivery. Birth weight was $2485 \mathrm{~g}$ ( $<5$ th centile). The amniotic fluid was heavily meconium stained and the baby started grunting immediately after birth. He was resuscitated but continued to have grunting and sternal retraction and required $80 \%$ oxygen. Examination showed proximal shortening of the limbs and bowing of the tibiae, limited extension of the elbows, camptodactyly, overlapping fingers, and mild micrognathia. The mouth appeared small.

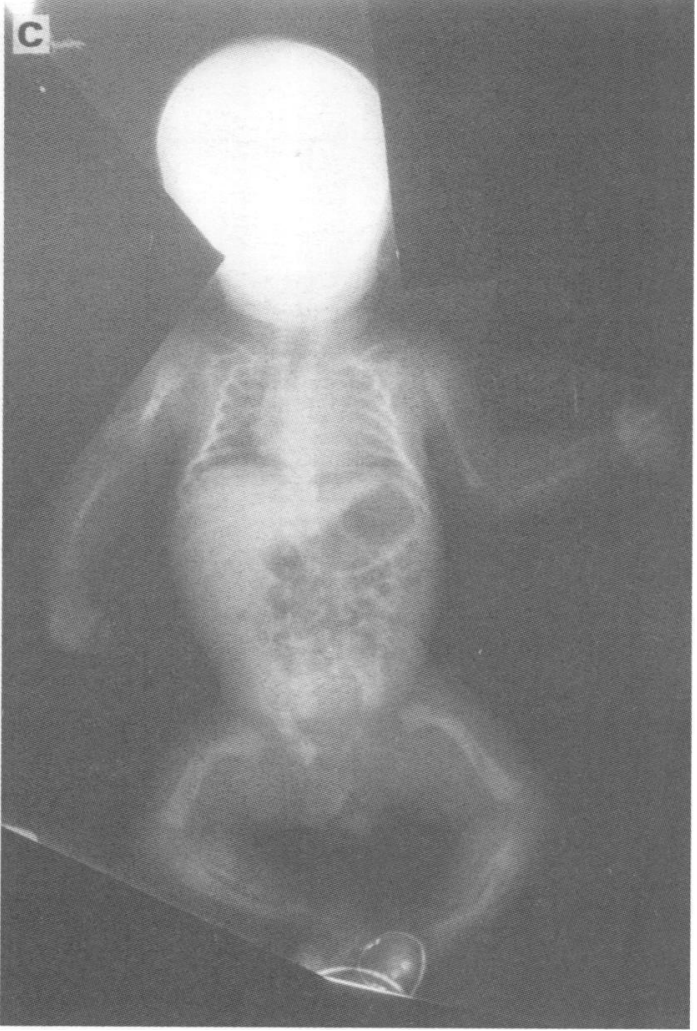

Figure $4(A, B)$ The facial appearance of case 1, family 2 crying and not crying. Note the pursed appearance of the mouth during crying, pectus excavatum, and camptodactyly with overlapping fingers. (C) Total $x$ ray of case 1, family 2 showing bowing of long bones and cortical thickening at the diaphyses.

In the two weeks of his stay in hospital, he could not be weaned off the oxygen. He developed unexplained fever. Investigations for possible sepsis were negative, and central fever was suspected.

Chromosome analysis, ultrasound of the kidney and brain, and echocardiography were normal. Skeletal survey at 5 months of age showed similar changes to the previous cases (fig $5 \mathrm{~A}$, B, table).

The baby had difficulty in feeding and required nasogastric tube feeding. He died at the age of 7 months after developing aspiration pneumonia.

\section{FAMILY 4}

The parents are first cousins of Omani origin and are of the same tribe as the third family (fig 1). The mother and father are 17 years old.

\section{Case 1 (III $\cdot 1)$}

This boy was the product of a normal pregnancy and delivery at 26 to 28 weeks' gestation. Birth weight was $1280 \mathrm{~g}$.

Multiple abnormalities were noted including small eyes, small mouth, arched palate, short, 


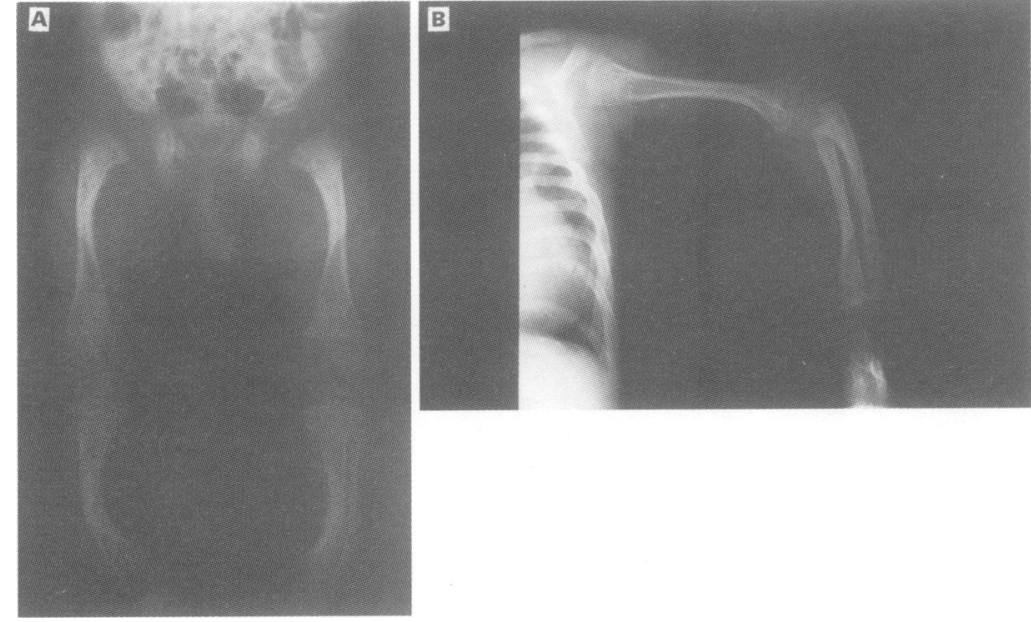

Figure $5 X$ ray of the long bones of the lower and upper limbs in III.6, family 3. Note the osteoporosis at the metaphyses and the cortical thickening.

bowed limbs, particularly the tibia, camptodactyly with overlapping fingers, limited extension of the elbows, and bilateral cryptorchidism.

The baby needed ventilatory support at birth. In the first few hours good oxygenation was achieved with $60 \%$ oxygen but later a dramatic increase was necessary to maintain proper oxygenation. Echocardiography showed the right aorta overriding the septum and possible subaortic ventricular defect with enlargement of the right ventricle.

The baby was fed by nasogastric tube and developed repeated attacks of aspiration pneumonia.

A few days before discharge he was noted to have thermal instability although blood culture
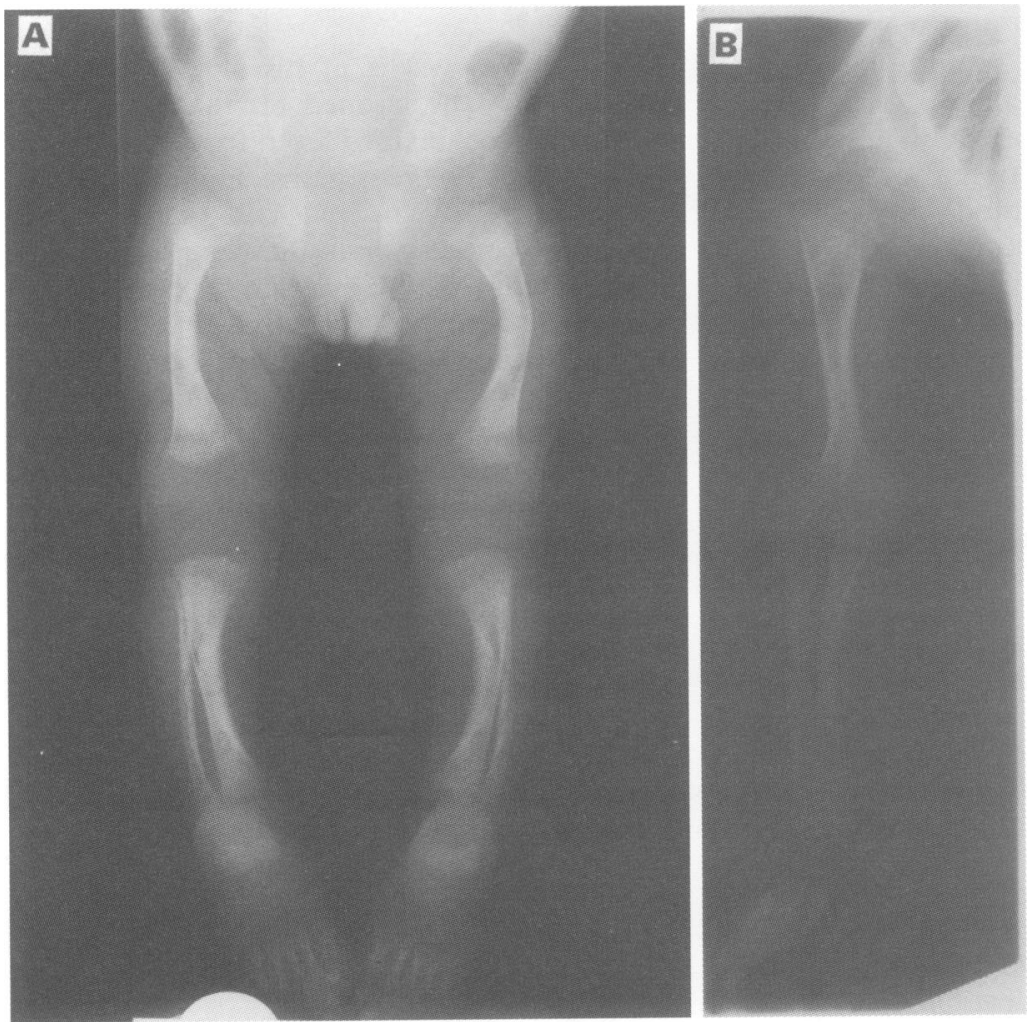

Figure $6(A, B) X$ ray of the long bones of the upper and lower limbs in III $\cdot 1$, family and other investigations for sepsis were normal. Skeletal survey at 4 months of age showed generalised osteoporosis, mainly at the metaphyses, which were widened and irregular, and bowing of the long bones with cortical thickening at the diaphyseal concavities, particularly affecting the lower limb bones and humeri (fig $6 \mathrm{~A}, \mathrm{~B}$, table).

He developed repeated attacks of aspiration pneumonia and died during one of these attacks.

\section{Case 2 (III·2)}

This boy was the second child of the couple. Pregnancy was normal and delivery at 31 weeks of gestation was normal. Birth weight was $1580 \mathrm{~g}$. He developed signs of RDS in the first hour after birth and needed ventilatory support for four days and oxygen in the incubator for another six days.

Examination and skeletal survey at birth showed exactly the same phenotype as his brother (table). Brain and renal ultrasound were normal.

Throughout his stay in hospital he was unable to suck and needed nasogastric tube feeding. He developed repeated attacks of aspiration pneumonia which led to his death at 1 month of age.

FAMILY 5

The parents are first cousins of Pakistani origin. The father is 37 and mother is 30 years old. Their first child died at the age of 3 months with similar abnormalities and inability to suck. There are three other normal children (fig 1). The mother's sister is married to the father's brother and their first child is said to be short with bowed limbs.

This pregnancy was normal and delivery at term of twin females was normal. Twin 1 had a birth weight of $3450 \mathrm{~g}$ (>50th centile) and head circumference of $35 \mathrm{~cm}$ (50th centile). She had the following dysmorphic features: short palpebral fissures, pursed appearance of the mouth during crying (fig $7 \mathrm{~A}, \mathrm{~b}$ ), bilateral camptodactyly with adducted thumbs, and mild ulnar deviation of the hands. There was limited extension of both elbows, mild bowing of the lower limbs, overlapping toes, and bilateral correctable positional talipes. The baby appeared stiff as a whole and had poor sucking and swallowing.

During her stay in the SCBU she was noted to have temperature instability but septic screening including urine, CSF, and blood culture were repeatedly negative. Brain ultrasound was normal. Chromosome analysis showed a normal female karyotype. She was discharged from SCBU on day 33 with nasogastric feeding.

Twin 2 had exactly the same features as her sister (table). All investigations were normal. Skeletal survey in the first month of life on both sisters showed increased bone density with flared metaphyses and mild bowing of the tibia (fig 7C, table).

They continued to have peaks of high temperature, needed nasogastric tube feeding, and 


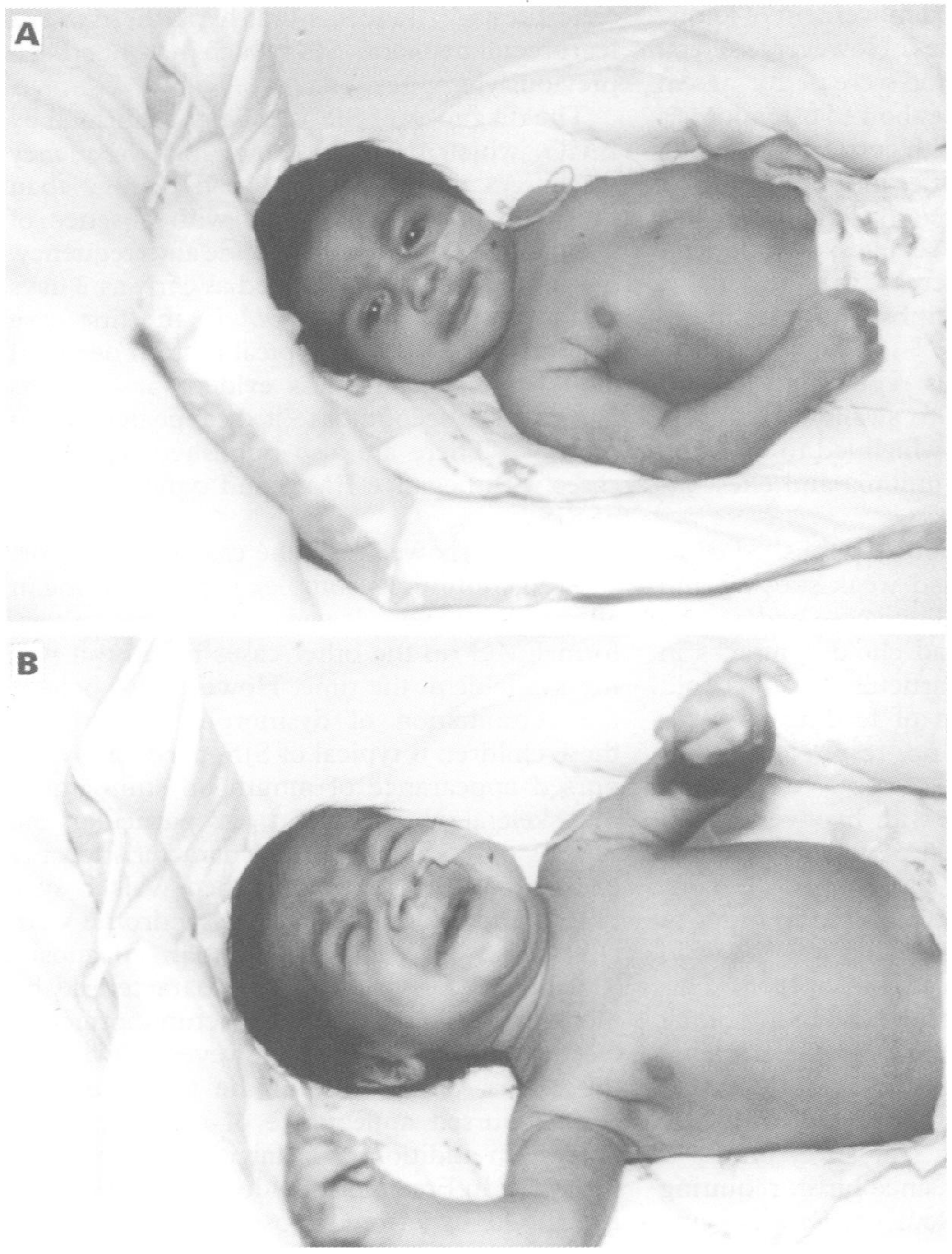

developed repeated attacks of aspiration. This led to the death of twin 2 at 4 months of age.

\section{Discussion}

SJS is a heterogeneous autosomal recessive syndrome of myotonia and bone dysplasia. More than 50 cases have been reported and two types have been recognised: the classical type with late infantile or childhood manifestation and a neonatal type where the features are usually apparent at birth or in the neonatal period.

We found 17 published cases of SJS with neonatal manifestation. ${ }^{1-11}$ These are reviewed and compared to the patients in this report. Neonatal manifestation of SJS includes facial dysmorphism, skeletal abnormalities, and feeding and respiratory problems.

In most of the previously reported cases, there was no detailed description of the faces in the neonatal period. Terms used to describe the face included "abnormal looking", "unusual face", and "cretinoid face". Only in the two cases of Hunziker et $a l^{7}$ was the typical pursed appearance of the mouth with the short palpebral fissures mentioned, while in the case of Farrell's et al the mouth was described as small.

In this report, five of the 11 cases had the typical facial appearance of SJS including the

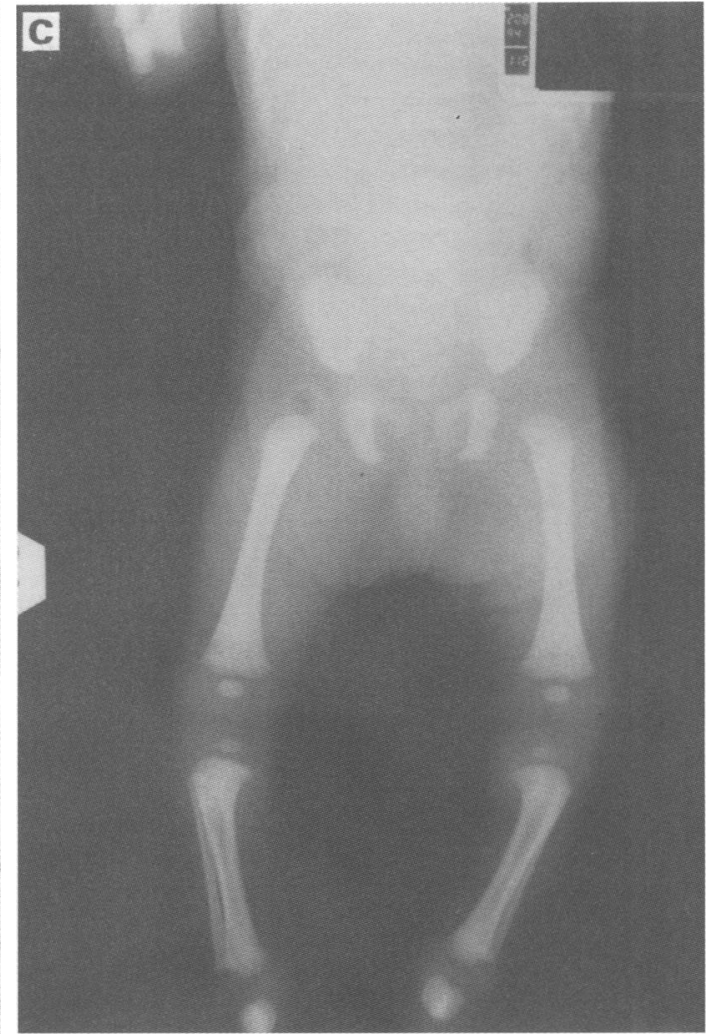

Figure 7 (A, B) Appearance of III.5, family 5 crying and not crying. (C) Increased bone density with widened metaphyses of the long bones in case III.5, family 5 .

short palpebral fissures and pursed appearance of the mouth. In three cases the mouth appeared small. It should be emphasised, however, that the typical mouth appearance was apparent only during crying in most cases and could readily have been missed. Other nonspecific features found in these children included micrognathia, high arched palate, and low set ears.

Skeletal abnormalities were present in all the children in this report. These included camptodactyly with overlapping fingers, bowing of the long bones, particularly the tibia (family 5 had milder bowing), and limited movement of the joints, particularly extension of the elbow. Limited abduction of the hip, talipes of the feet, and shortening of the limbs were present in some of them.

Skeletal $x$ rays showed identical changes in nine out of 11 cases in this report. These included generalised osteoporosis, particularly at the metaphyses of the long bones, bowing of the long bones, particularly the tibia, with cortical thickening at the diaphyseal concavity and widening of the metaphyses. In two cases (family 5) there was increased bone density and flared metaphyses of the long bones with mild bowing of the tibia.

Eleven of the 17 previously reported neonatal SJS cases had skeletal abnormalities at birth. ${ }^{1-79-11}$ These included limb shortening, 
bowing of the tibia, limited movement of joints, camptodactyly, and talipes. However, skeletal $x$ rays in the neonatal period were performed in four cases only. ${ }^{34711}$ These showed osteoporosis and bowing of the tibia with cortical thickening at the diaphyseal concavities in three cases. ${ }^{3711}$ In case 1 of Hunziker $e t a l^{7}$ skeletal $x$ rays at 3 months showed similar changes. In one case there was a generalised increase in bone density, wide sutures, and short limbs with wide metaphyses. ${ }^{4}$

All the children in this report had feeding difficulties with inability to swallow, requiring nasogastric tube feeding which led to repeated attacks of aspiration pneumonia and chest infections.

Only three of the previously published cases were reported to have had weak sucking and feeding difficulties ${ }^{279}$ and one case had a weak sucking reflex. ${ }^{4}$ Three had choking attacks in the neonatal period, particularly after cold drinks, with no mention of feeding or swallowing difficulties and no respiratory complications. $^{68}$

Respiratory difficulties with hypoventilation presenting in the neonatal period was present in eight out of the 11 cases in this report. High oxygen delivery was required in order to maintain adequate $\mathrm{Po}_{2}$ and this was followed by difficulty in weaning off the oxygen. This led to death in the neonatal period of five of the 11 children. Four cases survived the neonatal period but developed repeated chest infections resulting in death before 2 years of age. The remaining two cases are still alive; one is 3 months old, hospitalised since birth, requiring continuous oxygen supplementation, and one is 5 months old on nasogastric feeding with repeated attacks of aspiration pneumonia.

Respiratory distress after birth associated with hypoventilation requiring ventilatory support was present in the case of Farrell et al. ${ }^{4}$ This led eventually to death and necropsy showed hypertrophy of the muscles of the diaphragm, tongue, larynx, and perilaryngeal muscles. A milder degree of respiratory distress at birth was present in three other cases. ${ }^{2911}$ The first case of Hunziker et $a l^{7}$ died at 3 months after developing diarrhoea and dehydration and although there were no respiratory symptoms, necropsy showed severe interstitial and intraalveolar pneumonopathy. Their second case developed repeated episodes of aspiration but was alive at 10 months of age. ${ }^{7}$ Respiratory complications resulted in death in five other cases. $^{2911}$ This complication has been reported only in the neonatal type of SJS and is the cause of death in the majority of the cases. Although the precise pathophysiology of the complications is not known, contributing factors include hypertrophy of the muscles of the diaphragm, tongue, larynx, and perilaryngeal muscles, difficulty in swallowing leading to aspiration, and IgA deficiency reported in some patients. ${ }^{411}$

Seven of the cases in this report had attacks of unexplained hyperthermia and all relevant investigations for sepsis were negative. This feature was reported in two other cases. ${ }^{26}$ Two other cases had hyperthermia after anaesthesia ${ }^{610}$ It seems that hyperthermia is a feature of neonatal SJS which has not been previously emphasised.

The diagnosis of SJS is usually confirmed by EMG, which typically shows high frequency discharges more constant in frequency than those of classical myotonia with absence of waxing and waning in amplitude and frequency. These findings were reported as early as 2 days in the case of Farrell et al. ${ }^{4}$ In the first case of Hunziker et $a l^{7}{ }^{7}$ the typical pattern detected at 3 weeks became less evident at 6 weeks and in their second case it disappeared by 10 months. ${ }^{7}$ There are also published reports of cases with negative EMG and typical features of SJS. ${ }^{12}$

EMG at six weeks in one case in this report showed continuous complex repetitive firing in all muscles tested. It was not possible to perform EMG on the other cases because it was not available at the time. However, we believe the combination of dysmorphic features in these children is typical of SJS, particularly the pursed appearance of mouth on stimulation, the skeletal abnormalities, in particular the bowing of the tibia, and the characteristic $x$ ray changes of the bones. Marden-Walker syndrome and Freeman-Sheldon syndrome were considered in the differential diagnosis. Marden-Walker syndrome is characterised by blepharophimosis, joint contracture, immobile face, and small mouth. However, bowing of the bones, particularly of the tibia, and the typical pursed appearance of the mouth are absent. In addition, skeletal $x$ rays are usually normal. ${ }^{13}$ In Freeman-Sheldon syndrome there is marked hypertonia, absence of joint contractures, and the whistling appearance of the mouth is constant and not intermittent. There are also no $x$ ray changes or bowing of the bones. ${ }^{1415}$

It has been suggested that the neonatal manifestation of SJS indicates a more severe clinical course with a poor prognosis. However, some of the neonatal SJS cases reported previously had a similar course to the late onset type with no feeding or respiratory complications and normal survival. ${ }^{135810}$ Therefore, it appears that within the neonatal type of SJS there is a subgroup which manifests severe respiratory problems and has a poor prognosis. Identification of the molecular basis will help us to understand the clinical variability of this syndrome. Recently the SJS gene has been mapped to 1p34-p36.1 using homozygosity mapping in four inbred families of different ethnic backgrounds (three from Tunisia and one from South Africa). ${ }^{16}$ Similar studies are in progress to map the gene in our families.

We have recently reported a UAE family with three children affected with this type of SJS. ${ }^{11}$ This report brings the total number of children with neonatal SJS reported from the UAE to 14 from six families. This represents the largest review of this syndrome reported so far from one centre. Furthermore, all except two cases (one family) are of UAE or Omani origin and come from the $\mathrm{Al}$ Ain area (population 280000 ), giving a frequency of $1 / 23000$ in the general population (and much higher if only 
UAE and Omanis are considered since they constitute about 30 to $40 \%$ of the general population). This frequency is high for a recessive malformation syndrome and is comparable to other malformation syndromes identified as being common in the Arabs of Kuwait, such as multiple pterygium syndrome and Bardet-Biedl syndrome. ${ }^{1718}$

The authors thank Dr Robert Mueller for conducting the molecular studies on some of these families.

1 Beighton P. The Schwartz syndrome in Southern Africa. Clin Genet 1973;4:540-55.

2 Cao A, Cianchetti C, Calisti L, de Virgilis S, Ferelli A, Tangheron W. Schwartz-Jampel syndrome. $\mathcal{f}$ Neurol Sci 1978;35:175-87.

3 Fariello R, Meloff K, Murphy EG, Reilly BJ, Armstrong D. A case of Schwartz-Jampel syndrome with unusual muscle biopsy findings. Ann Neurol 1978;3:93-6.

4 Farrell SA, Davidson RG, Thorp P. Neonatal manifestations of Schwartz-Jampel syndrome. Am F Med Genet 1987;27: 799-805. 5 Ferguson DS, Young ID, Teoh R. Congenital myopathy Neurol 1981;23:237-42.

6 Fowler WM, Layler R, Taylor R, et al. The Schwartz-Jampel syndrome. F Neurol Sci 1974;22:127-46.
7 Hunziker URSA, Savoldelli G, Boltshauser E, Geidion A, Schinzel A. Prenatal diagnosis of Schwartz-Jampel syndrome with early manifestation. Prenat Diagn 1989;9. 127-31.

8 Huttenlocher PR, Landwirth J, Hanson V, Gallagher BB, Bensch K. Osteo-chondro-muscular dystrophy. A disorder manifested by multiple skeletal deformities, myotonia and manifested by multiple skeletal deformities, myotonia and
dystrophic changes in muscle. Pediatrics 1969;44:945-58.

dystrophic changes in muscle. Pediatrics 1969;44:945-58.
9 Rosignoli RF, Zanini F. Sindrome di Schwartz-Jampel. Description of a case with neonatal manifestation and review of the literature. Minerva Pediatr 1983;35:509-13.

10 Seay AR, Ziter FA. Malignant hyperpyrexia in a patient with Schwartz-Jampel syndrome. F Pediatr 1978;93:83-4.

11 Al-Gazali LI. The Schwartz-Jampel syndrome. Clin Dysmorphol 1993;2:47-54.

12 Moodley M, Moosa A. Chondrodystrophic myotonia (Schwartz-Jampel syndrome) in South African children. Neuropediatrics 1990;21:206-10.

13 Williams MS, Josephron KD, Wargowski DS. MardenWalker syndrome: a case report and a critical review of the literature. Clin Dysmorphol 1983;2:211-9.

14 Sanchez JM, Keminker CP. New evidence for genetic heterogeneity of the Freeman-Sheldon syndrome. Am $\mathcal{F} \mathrm{Med}$ geneity of the Freema

15 Antley RM, Uga N, Burzynski NJ. Diagnostic criteria for the whistling face syndrome. Birth Defects 1975;11:161-8.

16 Nicole S, Ben Hamida C, Beighton P, et al. Localization of the Schwartz-Jampel syndrome (SJS) locus to chromosome IP34-P36.1 by homozygosity mapping. Hum Mol Genet 1995;4:1633-6.

17 Farag TI, Teebi AS. High incidence of Bardet-Biedl syndrome among the Bedouin. Clin Genet 1989;36:463-5.

18 Teebi A, Daoud A Multiple pterygium syndrome: a relatively common disorder among Arabs. f Med Genet 1990; 27:791. 